\title{
Delayed matching-to-sample performance as a measure of human visuospatial working memory
}

\author{
WENDY V. PARR \\ Victoria University of Wellington, Wellington, New Zealand
}

(Murray J. White, Sponsor)

\begin{abstract}
The characteristics of the cognitive processes or systems involved in temporary maintenance of visual information are as yet unclear. In the present delayed matching procedure, subjects judged whether two visual patterns were identical under conditions in which patterns could vary in size and delay-interval length varied. To address the suggestion that a component of working memory is specialized for short-term visual storage, the type of distractor activity interpolated in the delay interval varied in terms of apparent differences in demands for working memory resources. Analyses of errors indicated that judgments of size disparity between two successively presented visual patterns were influenced by the length of the delay interval, by the specific processing activity interpolated in the delay, and by the temporal arrangement relating distractor activity and memory testing. Visual recognition was interfered with when memory testing occurred concurrently with the terminal processing of distractor activity, but not when testing occurred following termination of such distractor-activity processing. This result supports the view that a passive, visuospatial slave system-rather than central processing resources-is responsible for temporary maintenance of a visuospatial stimulus (Logie, Zucco, \& Baddeley, 1990). Data from delayed matching tasks may contribute to the theoretical development of a working memory system.
\end{abstract}

Tasks that require the temporary storage and manipulation of information are generally considered to involve working memory. Currently, the most frequently cited model of working memory is that of Baddeley (Baddeley, 1986; Baddeley \& Hitch, 1974). The model is assumed to comprise three components: a limited-capacity central executive that is involved in reasoning and decision making, and two independent slave systems - the articulatory loop involved in the storage and processing of verbal material, and the visuospatial sketchpad (VSSP), which performs a similar function for visuospatial material.

A number of tasks appear to involve temporary storage and manipulation of visuospatial information-for example, visual-imagery mnemonics, or the remembering of random square matrices (Phillips, 1974) or Chinese characters low in frequency and unlikely to have a verbal label known by subjects (Hue \& Erickson, 1988). Support for the idea that information can be maintained in a visual form also comes from a paradigm wherein comparisons in a memory task that can be made on the basis of physical appearance are faster than those that cannot. For example, Parks, Kroll, Salzberg, and Parkinson (1972) showed that matches of physically identical stimuli (e.g., "A" to " $A$ ") after an 8-sec delay were relatively faster than

This research was supported by a grant from Victoria University of Wellington Internal Grants Committee (495A) to Wendy Parr. I thank Judi Lapsley for assistance with data collection. Correspondence should be addressed to Wendy V. Parr, Victoria University of Wellington, P.O. Box 600, Wellington, New Zealand. matches with stimuli that were the same in name but different in case (e.g., "A" to "a"), suggesting that visual traces were maintained for at least 8 sec.

The existence of a system, such as Baddeley's articulatory loop, that is specialized for processing and temporary storage of verbal material is relatively well established (Avons \& Phillips, 1987; Baddeley, 1986; Logie, 1989). The existence of a similar specialized system for processing visuospatial information is a more contentious issue. Two views of whether or not a VSSP is a necessary component of an adequate working memory model have dominated the literature.

Baddeley and colleagues (Baddeley \& Lieberman, 1980; Logie, 1986; Logie, Zucco, \& Baddeley, 1990) have provided data from a range of memory tasks and intervening tasks, which they argue support the existence of a separate system specialized for storage and manipulation of visuospatial information. Recently, using a span task, Logie et al. (1990) found a double dissociation: a letter span task was more disrupted by concurrent arithmetic than by a visuospatial task, whereas the visual matrix task showed greater disruption when the concurrent activity was visuospatial rather than arithmetic. The assumption behind these studies is that the slave systems can be dissociated from each other by employing interference activities specifically targeted at a given slave system. Consequently, there has been much concern with modality, but less with the specific details of memory tasks and intervening tasks, including assumed involvement of central resources in these often complex tasks. 
Phillips and Christie (1977a, 1977b), on the other hand, have reported findings that conflict with the view that a separate, independent system is necessarily involved in the temporary storage of visuospatial information (Avons \& Phillips, 1980, 1987; Phillips, 1974; Phillips \& Christie, 1977a, 1977b). Using a paradigm involving recognition memory for visually presented matrices, in which the matrices typically have half their cells filled, and one cell is changed at random between stimulus presentation and recognition test, these researchers have obtained data from which they have argued that the processing and storage of short-term visual information may rely on a central resource pool, not a separate system, so that any distractor task that is "mentally demanding" (Avons \& Phillips, 1980, p. 407) will compete for resources. For example, visual memory was impaired when subjects were required to add aurally presented digits. They argue that the most crucial factor in the disruption of visuospatial working memory is not the visual similarity of the to-beremembered event and the intervening task, but rather the extent to which the interpolated task requires central processing capacity.

Development of a coherent view of the characteristics of visuospatial working memory has been complicated by the use of different paradigms (e.g., Brown-Peterson tasks; span tasks) and tasks varying in complexity. Task difficulty is a particularly important issue, and the current empirical and theoretical contradictions concerning the need to posit a separate system for temporary maintenance of visuospatial information may be addressed by systematically modifying relevant variables within a particular task to determine precisely which aspects are responsible for any interference caused. The delayed matching procedure could serve such a purpose. The standard task lends itself to substantial variation, including modification of temporal variables and of the nature of the stimuli used in memory and intervening tasks, while important variables such as the mode of responding, and hence, dependent measures, are held constant. Delayed matching tasks have been shown to be sensitive to human amnesia (e.g., in Korsakoff patients) and to recognition performance in people with dementia of the Alzheimer type, and these tasks have advantages over more complex tasks, in that subjects have little difficulty in understanding the task instructions.

In the present study, the selectivity of interference in working memory was investigated in a delayed matching procedure. It was specifically aimed at the investigation of whether or not distractor tasks with minimal requirements on visuospatial processing, but varying in demands on central processing resources, would differentially affect memory for a visual stimulus.

A second purpose was to attempt to test a prediction from Baddeley's working memory model concerning the involvement of central executive resources in maintenance of a visual stimulus over the delay interval-that is, to investigate the need for a separate system for visuospatial information. Critical to distinguishing between Baddeley's view and that of Phillips and Christie is a delay-interval manipulation in which only part of the delay is filled with intervening activity. This would allow recognition performance to be measured with concurrent activity, as well as following the termination of such activity. If the visual stimulus is maintained in a separate store, intervening activity with low demands on visual processing should affect performance only when memory testing occurs concurrently with intervening activity draining centralprocessing resources. No studies appear to have measured primary task performance beyond the divided-attention component of the procedure.

\section{METHOD}

\section{Subjects}

There were 40 subjects -19 females and 21 males, between 17 and 59 years of age. They were staff and students of Victoria University of Wellington, New Zealand, and they were paid to participate in the experiment. All had normal or corrected-to-normal vision.

\section{Materials}

The experiment was conducted and responses were recorded on a Commodore PC 40, Series III microcomputer, with task instructions and visual stimuli presented on a Hewlett-Packard videographics color monitor.

The stimuli were squares varying in size. Eight stimuli were used, varying from 24 to $36 \mathrm{~mm}(24,26,27.5,29,30.5,32.5,34$, and $36 \mathrm{~mm})$ on a side. In selecting stimuli that would encourage visual coding and maintenance, it was assumed that variations in the size of a square would not lend themselves readily to a verbal label (cf. Kubovy \& Podgorny, 1981). A simple stimulus was chosen for two reasons. First, it could be assumed that the capacity of a visual store would not be exceeded (Frick, 1988). Second, a problem with many tasks used previously (e.g., versions of Brooks's, 1967, 1968, tasks) is that they are likely to make demands on a central resource pool, irrespective of whether a separate system exists for specialized processing, resulting in complicating theoretical interpretations. The memory task used in the current study could be assumed low in its demands on a central executive, but high in its demands on a specialized visual processor.

\section{Design and Procedure}

The design was a $4 \times 3$ within-subject factorial, with four levels of delay interval and three levels of condition.

The experiment was conducted under normal room illumination. Each session involved 9 practice trials followed by 96 experimental trials, with each session's trials in a unique random order. The subjects were tested individually, and the experimenter was present throughout.

In the successive matching-to-sample task, each trial involved presentation of a fixation point for $1 \mathrm{sec}$ in the central location of the monitor. A sample stimulus was then presented in the same location for $1 \mathrm{sec}$. The sample and test stimuli were relatively large in comparison with the fixation point. After a variable delay interval $(0,5,10$, or $15 \mathrm{sec})$, each occurring with equal probability, a test stimulus was presented in the same location as that of the previously seen sample. The subject's task was to decide whether the stimulus was exactly the same as the previously presented sample, and to press one of the designated response keys accordingly. For half of the subjects, the response "same" was made with the index finger of the right hand, and the response "different" with the index finger of the left hand. For the remainder of the subjects, these responses were reversed. A 3-sec intertrial interval separated consecutive trials in a session. Feedback in the form of the word "correct" or "error" was displayed centrally on the monitor for $1 \mathrm{sec}$ after the subject's response had been made. The remaining $2 \mathrm{sec}$ of the intertrial interval were blank.

There were three conditions that differed in terms of activity interpolated in the delay interval. Other than when a 0 -sec delay occurred, a word appeared on the monitor for $1 \mathrm{sec}$ at the fixation-point location, 
Table 1

Mean Proportions Correct (With Standard Deviations) As a Function of Condition and Delay (in Seconds)

\begin{tabular}{|c|c|c|c|c|c|c|}
\hline \multirow[b]{2}{*}{ Delay } & \multicolumn{2}{|c|}{ Control } & \multicolumn{2}{|c|}{ Passive } & \multicolumn{2}{|c|}{ Active } \\
\hline & $M$ & $S D$ & $M$ & $S D$ & $M$ & $S D$ \\
\hline 0 & 0.90 & 0.121 & 0.86 & 0.135 & 0.87 & 0.137 \\
\hline 5 & 0.74 & 0.148 & 0.68 & 0.157 & 0.63 & 0.179 \\
\hline 10 & 0.70 & 0.157 & 0.75 & 0.128 & 0.69 & 0.155 \\
\hline 15 & 0.70 & 0.167 & 0.72 & 0.128 & 0.63 & 0.199 \\
\hline
\end{tabular}

$.5 \mathrm{sec}$ after sample-stimulus offset in all conditions. The three conditions included a control condition and two "interference" conditions, in which distractor tasks were used. In the control condition, the word "blank" appeared $.5 \mathrm{sec}$ after sample offset, indicating to the subject that no activity would occur during the delay. In the other two conditions, the word that appeared was followed by a string of five digits, presented visually at the fixation point at a rate of two per second. In the passive condition, the word "read" occurred, indicating that the five subsequently presented digits were to be read aloud to the experimenter. The remainder of the delay interval (10- and 15 -sec delays) was free of interpolated activity. In the active condition, the word "add" appeared after sample offset and indicated that the five digits were to be added together, with their sum stated aloud to the experimenter. Again, the remainder of the longer delay intervals was free of interpolated activity. The digits were presented successively at a rate of two per second, so that the final digit in the distractor activity occurred $4 \mathrm{sec}$ into the delay interval. The distractor tasks had identical visual components, but the active (digit-adding) task was assumed to require central processing resources, whereas the passive (digit-recognition) task was assumed on the basis of previous research to require the articulatory loop but to make minimal demands on central processing resources. The control condition, in which there was no concurrent activity, served as a baseline.

The combination of eight stimuli and four delay intervals gave 32 trials in each of the three conditions. A random generator selected combinations of same/different comparisons with equal probability for each subject, with the constraint that no more than three "same" or "different" responses occur on consecutive trials. Combinations of samples and delays were selected at random, with the constraint that no more than three consecutive trials include the same delay-interval length or the same sample stimulus. The type of interpolated activity on any particular trial was not known until after sample presentation, and the length of the delay interval was not known until presentation of the test stimulus. A session lasted approximately $35 \mathrm{~min}$.

\section{RESULTS}

The proportion of correct responses (correct/total responses) for each subject (eight trials with each of the 12 combinations of condition and delay) was calculated. Table 1 shows the mean proportions correct (PC) for each delay interval in each condition. Performance is highest overall in the control condition (mean PC $=76.01$ ) and lowest in the active condition (mean PC $=70.47$ ). In both interference conditions, performance is disrupted at the 5-sec delay, when the subject had to respond to the delayed matching test concurrently with completion of distractor activity (the last distractor digit having been presented $4 \mathrm{sec}$ into the delay interval). These results are summarized in Figure 1, in which delayed matching performance is plotted as a function of delay interval and of whether the memory test occurred concurrently with terminal processing of the distractor task, or subsequent to it. Control condition data are used in both subplots for comparative purposes.

The 480 proportions correct $(4$ delays $\times 3$ conditions $\times 40$ subjects), each based on eight trials conducted with the combinations, were subjected to a $4 \times 3$ analysis of variance for repeated measures on both factors. There was a main effect of delay $[F(3,117)=47.38, p<.01]$. There was also a main effect of condition $[F(2,78)=6.27$, $p<.01]$. The delay $\times$ condition interaction failed to reach significance. When the data were submitted to an arc-sin transformation, a similar result was found [for condition, $F(2,78)=5.72, p<.01$; for delay, $F(3,117)=$ $55.64, p<.01]$.

A post hoc comparison by means of Duncan's multiple range test $(\alpha=.05)$ on scores subjected to a one-way analysis of variance at each delay interval showed that the active condition differed from the control condition at the 5-sec delay, but the test was ambiguous with respect to the passive condition. No difference was found between conditions at the 10-sec delay. At the 15-sec delay, the active condition differed from the passive condition.
With Interpolated Activity

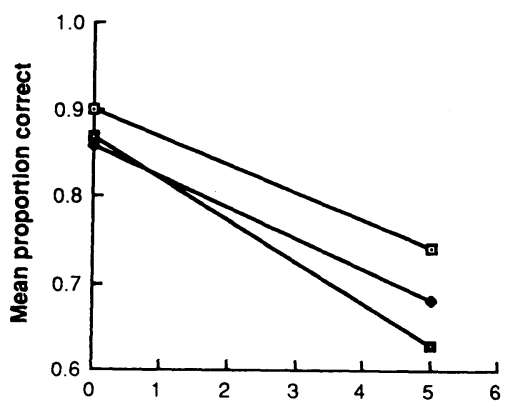

Without Interpolated Activity

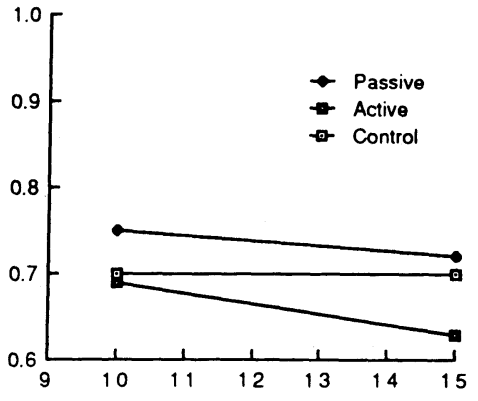

Retention Interval (sec)

Figure 1. Delayed matching performance as a function of retention interval with and without interpolated activity at the time of the memory test. 


\section{DISCUSSION}

These results show short-term retention of visual information, as measured by the delayed matching task, to be differentially affected by digitadding and digit-recognition distractor tasks. Poorer overall performance on the active (digit-adding) task suggests the involvement of central processing resources in the maintenance of a visual stimulus over the delay interval, a finding more in keeping with Phillips and Christie's (1977a, 1977b) conclusions than with those of Baddeley (1986) and Logie et al. (1990).

However, when performance is looked at separately in terms of whether visual recognition was tested concurrently with termination of distractor activity (5-sec delay testing), or subsequent to termination of distractor activity (10- and 15-sec delays), the results are more in keeping with Baddeley's working memory model. The critical result, in terms of the two theoretical positions previously outlined, involves the greater disruption at the 5 -sec delay by a task requiring the adding of digits than by a task requiring the recognition of digits, and the subsequent improvement in performance at the 10-sec delay in both interference conditions. Baddeley's model predicts recognition to be affected only when testing occurs concurrently with interpolated activity draining central resources (i.e., in the active task at the 5-sec delay). Predictions are less clear with respect to the passive task, but if it is influential at all, again it should have its effect at the 5-sec delay only. On the other hand, Phillips and Christie's model predicts the disruption of performance at the 5-sec delay to be carried through to the longer delays, since central processing resources are assumed to be necessary for the maintenance of short-term visual information. The current result clearly does not support this, although a decline in performance at the 15-sec delay in the active condition complicates the interpretation. Two factors to consider with respect to the latter point are the large amount of variability in performance at the longer delays in the active condition (see Table 1) and the confounding with delay-interval length inherent in the current methodology.

Disruption of delayed matching at the 5-sec delay but an absence of a similar effect of condition at the 10-sec delay suggests that maintenance of a visual stimulus is not disrupted by either interference condition unless recognition is tested while central processing resources are also involved in completing a distractor task. The current result therefore supports a working memory model in which a store, separate from the central executive, maintains a visual stimulus, making minimal demands on central resources until time of recognition test. Morris (1987) has proposed that the central executive in Baddeley's model is involved in active encoding of material into the VSSP, but that it is "uncoupled" from the VSSP during maintenance rehearsal and freed up for other tasks until testing occurs. In the task used in the present study, central executive involvement should be consistent involvement at each delay interval (i.e., the testing situation remains constant), so that the delay-interval manipulation allows assessment of the maintained visuospatial information with central executive involvement held constant. Theoretically, then, the effect of the active and passive distractor tasks can be ascertained.

Improvement of performance following cessation of distractor activity (i.e., at the 10-sec delay) for both interference conditions implicates the "divided attention" component of the task in the poor performance demonstrated in many paradigms. When Phillips and Christie found an effect of adding visually and aurally presented digits on performance of their visual matrix task, they measured recognition memory at one delay only, at $1.25 \mathrm{sec}$ after presentation of the final distractor digit. Hence, finding that any mentally demanding task, irrespective of modality, disrupted visual memory performance may reflect the central processing component, and it leaves open the question of the necessity of postulating a separate visual store utilized for temporary maintenance of visual information. Logie et al. (1990) have argued that the small impairment on visual-span performance by a concurrent arithmetic task reflects the general disruption from concurrent activity, but that the more specific disruption by a same-modality task reflects the operation of a specialized processor.
In the current study, the change in accuracy over the delays in the control condition indicates that the delayed matching-to-sample procedure is suitable for the investigation of the temporary maintenance of visual information in human subjects. Control-condition performance suggests that the decay or loss of visual memory is most rapid in the first few seconds of the delay interval (cf. Peterson \& Peterson, 1959). However, the development of a version of the procedure that is less prone to the effects of individual differences among subjects is desirable and is currently under investigation.

In summary, the current result supports the concept of a specialized system in working memory for temporary storage of visual information. Data from human delayed matching tasks have the potential to contribute toward the development of a coherent account of the nature of working memory.

\section{REFERENCES}

Avons, S. E., Phillips, W. A. (1980). Visualization and memorization as a function of display time and poststimulus processing time. Journal of Experimental Psychology: Human Learning \& Memory, 6, 407-420.

Avons, S. E., \& PHILlips, W. A. (1987). Representation of matrix patterns in long- and short-term visual memory. Acta Psychologica, 65, 227-246.

Baddeley, A. D. (1986). Working memory. Oxford: Oxford University Press.

BADDELEY, A. D., \& Hitch, G. J. (1974). Working memory. In G. Bower (Ed.), The psychology of learning and motivation (Vol. 8, pp. 47-90). London: Academic Press.

Baddeley, A. D., Lieberman, K. (1980). Spatial working memory. In R. Nickerson (Ed.), Attention and performance VIII (pp. 521539). Hillsdale, NJ: Erlbaum.

Brooks, L. R. (1967). The suppression of visualization by reading. Quarterly Journal of Experimental Psychology, 19, 289-299.

Brooks, L. R. (1968). Spatial and verbal components of the act of recall. Quarterly Journal of Experimental Psychology, 10, 349-368.

Frick, R. W. (1988). Issues of representation and limited capacity in the visuospatial sketchpad. British Journal of Psychology, 79, 289-308.

HuE, C.-W., ERICKSON, J. R. (1988). Short-term memory for Chinese characters and radicals. Memory \& Cognition, 16, 196-205.

Kubovy, M., \& Podgorny, P. (1981). Does pattern matching require the normalization of size and orientation? Perception \& Psychophysics, 30, 24-28.

LOGIE, R. H. (1986). Visuo-spatial processing in working memory. Quarterly Journal of Experimental Psychology, 38A, 229-247.

LOGIE, R. H. (1989). Characteristics of visual short-term memory. European Journal of Cognitive Psychology, 1, 275-284.

LoGIE, R. H., Zucco, G. M., B BADELEY, A. D. (1990). Interference with visual short-term memory. Acta Psychologica, 75, 55-74.

MoRRIS, N. (1987). Exploring the visuo-spatial scratch pad. Quarterly Journal of Experimental Psychology, 39A, 409-430.

Parks, T. E., Kroll, N. E. A., Salzberg, P. M., \& Parkinson, S. R. (1972). Persistence of visual memory as indicated by decision time in a matching task. Journal of Experimental Psychology, 92, 437-438.

Peterson, L. R., \& Peterson, M. J. (1959). Short-term retention of individual verbal items. Journal of Experimental Psychology, 58, 193-198.

PhILlips, W. A. (1974). On the distinction between sensory storage and short-term visual memory. Perception \& Psychophysics, 16, 283-290.

Phillips, W. A., \& Christie, D. F. M. (1977a). Components of visual memory. Quarterly Journal of Experimental Psychology, 29, 117-133.

Phillips, W. A., \& Christie, D. F. M. (1977b). Interference with visualization. Quarterly Journal of Experimental Psychology, 29, 637-650.

(Manuscript received February 25, 1992.) 